\title{
Contribution of p38 MAPK to the Ameliorating Effect of Enriched Environment on the Cognitive Deficits Induced by Chronic Cerebral Hypoperfusion
}

\author{
Yu-Wang Li Qing-Yun Li Jin-Hua Wang Xiao-Lin Xu \\ Department of Neurology, Tianjin Huanhu Hospital, Tianjin, PR China
}

\section{Key Words}

Enriched environment • p38 MAPK • Vascular dementia

\begin{abstract}
Background/Aims: An enriched environment (EE) ameliorates learning and memory impairments induced by chronic cerebral hypoperfusion, and the p38 mitogen-activated protein kinase (p38 MAPK) signaling pathway exerts both beneficial and deleterious effects on the nervous system during the progression of ischemia. Methods: The present study investigated whether p38 MAPK participates in the process by which EE exposure ameliorates the cognitive deficits induced by chronic cerebral hypoperfusion. Results: EE exposure significantly enhanced the cognitive performance of vascular dementia (VD) model rats, and p38 MAPK protein decreased in parallel with cognitive improvements. Inhibition of p38 MAPK function by its selective inhibitor SB203580 improved the cognition index of VD rats and upregulated p38 MAPK expression with p38 MAPK antisense oligodeoxynucleotides. This impaired cognition in VD rats could not be rescued by EE exposure. Conclusion: P38 MAPK participates in the process by which EE exposure ameliorates cognitive deficits induced by chronic cerebral hypoperfusion.

\section{Introduction}

Vascular dementia (VD) is the second most common type of dementia-related illness after Alzheimer's disease. Rats with chronic cerebral hypoperfusion induced by permanent bilateral common carotid artery occlusion (BCCAO) have been widely used to explore the pathological mechanisms of VD and to develop new treatment options [1]. The hippocampus Y.-W. Li and Q.-Y. Li are co-first authors. 


\section{Cellular Physiology Cell Physiol Biochem 2016;40:549-557 \begin{tabular}{l|l|l} 
DOI: 10.1159/000452568 & (c) 2016 The Author(s). Published by S. Karger AG, Basel \\
www.karger.com/cpb
\end{tabular} \\ Li et al.: p38 MAPK in the Improvement of Cognitive Deficits by EE}

is a brain structure that plays an important role in learning and memory, and the CA1 within this region is the most susceptible to insult hypoxia or ischemia [2]. Studies have shown that activation of the p38 mitogen-activated protein kinase (p38 MAPK) signaling pathway by hypoxia may initiate neuronal apoptosis, leading to the functional deficits of VD [3].

Enriched environment (EE) exposure ameliorates spatial memory impairment after cerebral ischemia $[4,5]$ and has emerged as a powerful condition able to induce changes at the cellular, molecular and behavioral levels of the hippocampus [6]. In addition, EEs restore long-term potentiation (LTP) and spatial memory impairment induced by chronic cerebral hypoperfusion [7]. Furthermore, a study using Ras-GRF knockout mice found that EE exposure promoted LTP via a p38-dependent signaling pathway [8].

The cognitive function improved by EE was assessed by Morris water maze and parameters of the p38 MAPK pathway in BCCAO model rats.

\section{Materials and Methods}

\section{Animals}

Male Wistar rats (3 months) provided by the Experimental Animal Center of Tianjin medical University were used in this study. Animals were housed on a 12-h light/12-h dark cycle with ad libitum access to food and water. All experiments conformed to the guidelines for the care and use of laboratory animals.

\section{BCCAO and sham operation}

Animals were anesthetized by intraperitoneal injection of a $10 \%$ chloral hydrate solution at 0.35 $\mathrm{ml} / 100 \mathrm{~g}$ body weight. A midline incision was performed to expose both common carotid arteries, which were then tightly double-ligated using silk sutures. In addition, control rats were subjected to a sham operation in which they underwent the same procedure without BCCAO.

\section{Animal groups and process}

The rats were randomly assigned to the following groups: 1 . Sham group; 2 . Sham + dimethyl sulfoxide (DMSO) group; 3. Sham + artificial cerebrospinal fluid (ACSF) group; 4. VD group; 5. VD + DMSO group; 6. VD + ACSF group; 7. VD + EE group; 8. VD + SB203580 group; and 9. VD + antisense oligodeoxynucleotides (S-ODNs) + EE group. The VD model rats were established by BCCAO. For animals of the sham group, the bilateral carotid artery was separated using the same methods but without BCCAO. The VD + EE group included rats that underwent BCCAO and then experienced EE for 1 month. After recovery from BCCAO, the animals of the VD+SB203580 group received intracerebroventricular (ICV) injections of SB203580 (15 $\mu \mathrm{L}$

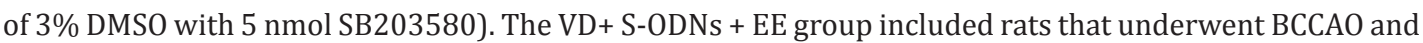
received ICV injections of S-ODNs (15 $\mu \mathrm{L}$ ACSF with $15 \mathrm{nmol} \mathrm{S-ODNs}$ ) and then experienced EE for 1 month. The Sham + DMSO and VD + DMSO groups included sham rats and VD rats that received ICV injections of $15 \mu \mathrm{L} 3 \%$ DMSO. The Sham + ACSF and VD + ACSF groups included sham rats and VD rats that received ICV injections of $15 \mu \mathrm{L}$ ACSF. Except for the VD + EE and VD + S-ODNs + EE groups, all animals were housed in standard environments. One month after the animal model operation, all rats were examined using the Morris water maze to assess spatial learning and memory ability. After the behavior test, the animals were euthanized, and tissues were prepared for Western blot assays and immunohistochemistry evaluations of p-p38 MAPK expression.

\section{Implantation of cannula}

A cannula was first implanted into the right cerebral ventricle. The heads of the rats were secured in a stereoscopic frame (ASI Instrument Company, USA) under halothane anesthesia and local anesthesia with $1 \%$ procaine solution. According to the atlas of Paxinos and Watson [9], a hole was made on the parietal bone ( $0.8 \mathrm{~mm}$ caudal to the bregma and $1.5 \mathrm{~mm}$ lateral to the midline) using a high-speed dental drill (OSADA Company, USA). After the dura was carefully incised, a cannula and its stylet (Reward InstrumentCompany, China) were inserted vertically into the lateral cerebral ventricle (3.8 $\mathrm{mm}$ ventral to the surface) and fixed to the skull with dental cement. Implantation of the cannula was performed 7 days before the BCCAO or sham operation. 


\section{Cellular Physiology Cell Physiol Biochem 2016;40:549-557

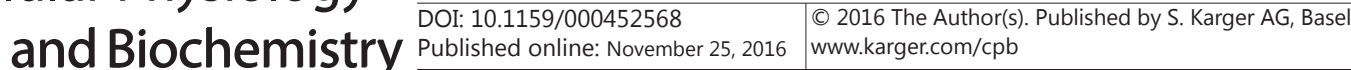 \\ Li et al.: p38 MAPK in the Improvement of Cognitive Deficits by EE}

\section{ICV Injection}

The stainless steel inner core of the implanted guide cannula was removed, and a needle connected with a microsyringe was inserted into the guide cannula. According to the design of the experiment, a 15 $\mu \mathrm{L}$ SB203580 solution or its solvent (3\% DMSO) or p38 MAPK S-ODN solution or its solvent (ACSF) was injected over a 15-min period. The needle was kept in place for an additional $10 \mathrm{~min}$ after the injection to prevent overflow of the injected solution, and then the cannula was sealed by the stainless steel inner core. During the entire process, the rats were kept awake and calm. ICV injections were administered on the day before operation, the day of the operation, and the day after the operation for a total of 3 administrations.

S-ODNS

The sequence of p38 MAPK S-ODNs was 5' - ATGTCGCAGGAAAGGCCC-3' [10]. To increase the stability of S-ODNs, all the bases were modified by sulfo-phosphorylation.

\section{Enriched environment}

The enriched environment cages $(50 \mathrm{~cm} \times 40 \mathrm{~cm} \times 40 \mathrm{~cm}, 5$ animals per cage) consisted of a variety of objects such as toys, plastic tunnels, climbing ladders, running wheels and stairs, which were added, removed, or changed each week. The standard environment cages $(50 \mathrm{~cm} \times 40 \mathrm{~cm} \times 40 \mathrm{~cm}, 5$ animals per cage) did not contain objects.

\section{Morris Water Maze Test}

The Morris water maze setup consisted of a circular pool (diameter: $150 \mathrm{~cm}$, height: $50 \mathrm{~cm}$ ) with a hidden platform $2 \mathrm{~cm}$ beneath the water. The rats were trained for 5 consecutive days, followed by the probe trial on day 6. The rats were released into the water in one of four quadrants of the pool. The order of the quadrants was changed daily in a random manner. The rats were trained four times per day (60 sec/ trial). The time required for the rats to find the hidden platform was recorded as the escape latency. After swimming for $60 \mathrm{sec}$, they were allowed to stay on the platform for 10 second before the next swim trial. Single probe trials to test reference memory were conducted on day 6. Rats were released at a random start position and were allowed to swim for $60 \mathrm{sec}$ in the absence of the platform. The time spent in the target quadrant was used to evaluate the spatial memory ability of the rats. The swimming speed was also recorded, and we found that the velocity $(\mathrm{cm} / \mathrm{m})$ of the rats did not significantly differ among groups $(P=$ $0.574, \mathrm{~F}=0.731$, Sham: $17.9 \pm 1.62$; VD: $18.0 \pm 1.71$; VD + EE: $17.3 \pm 1.63$; VD + SB202190: $18.2 \pm 1.75 ; \mathrm{VD}$ + S-ODNs + EE: $17.3 \pm 1.86, \mathrm{n}=12$ each group).

\section{Western Blot Analysis for p-p38 MAPK}

The hippocampal CA1 subfield was isolated and homogenized in lysis buffer [11]. The homogenates were centrifuged at $12,000 \times \mathrm{g}$ for $5 \mathrm{~min}$ at $4^{\circ} \mathrm{C}$, and the supernatant was retained. The protein concentration of the supernatant was determined using the Lowry method. Sixty milligrams of protein of each sample mixed with loading buffer was loaded in each lane. The samples were electrophoresed on a 10\% SDSpolyacrylamide gel and transferred to a PVDF membrane at $100 \mathrm{~V}$ for $3 \mathrm{~h}$. Nonspecific binding was blocked in Tris-buffered saline mixed with Tween-20 (TTBS) containing 5\% skim milk for $60 \mathrm{~min}$. The membranes were subsequently incubated with primary antibodies including rabbit anti-p-p38 MAPK monoclonal antibody (1:500, Cell Signaling Technology) or mouse anti- $\beta$-actin polyclonal antibody $\left(1: 1000\right.$, Santa Cruz) at $4^{\circ} \mathrm{C}$ for $24 \mathrm{~h}$. After washing with TPBS, the membranes were incubated at $37^{\circ} \mathrm{C}$ for $1 \mathrm{~h}$ with secondary antibodies to either IRDye700DX-conjugated biotin-labeled anti-rabbit IgG antibody (1:5000, Rockland company) for p-p38 MAPK or IRDye800CW-conjugated anti-mouse IgG antibody (1:5000, Rockland Company) for $\beta$-actin followed by washing with TPBS. The blots for p-p38 MAPK and $\beta$-actin were detected using an Odyssey Fc Imager, and the integrated optical density (IOD) values were obtained. The results are presented as the IOD ratio of p-p38 MAPK and $\beta$-actin.

\section{Immunohistochemistry for p-p38 MAPK}

The immunohistochemistry methods were according to Sun et al. [12]. The animals were anesthetized with $10 \%$ chloral hydrate and perfused through the ascending aorta with $150 \mathrm{ml}$ normal saline (NS) (0$4^{\circ} \mathrm{C}$ ) followed by $200 \mathrm{ml}$ of $4 \%$ paraformaldehyde $\left(0-4^{\circ} \mathrm{C}\right)$. The brain was then removed, and a $3-\mathrm{mm}$ brain slice including the bilateral hippocampus was excised coronally behind the optic chiasm. The embedded 
brain tissues were sectioned into 5-6 $\mu \mathrm{m}$-thick sections for immunohistochemistry. The sections were deparaffinized in xylene and rehydrated by passage through a decreasing series of ethanol solutions, followed by washing twice with water. The sections were then incubated in $3 \% \mathrm{H}_{2} \mathrm{O}_{2}$ for $15 \mathrm{~min}$ at room temperature to quench the endogenous peroxide. The samples were then incubated in antibodies against p-p38 MAPK (rabbit, monoclonal antibody, Cell Signaling) diluted at 1:120 overnight at $4^{\circ} \mathrm{C}$ after pre-incubation in $10 \%$ normal goat serum for $1 \mathrm{~h}$. The samples were washed with PBS, and secondary antibody (Goat anti-rabbit IgG, Santa Cruz) was then added to the slides and incubated for $50 \mathrm{~min}$ at $37^{\circ} \mathrm{C}$. After washing with PBS, the sections were developed with 3, 3-diaminobenzidine-tetrachloride- H2O2 (DAB-H2O2). Sections that were used for comparison were stained together and developed for the same amount of time. Omission of the primary antibodies in a subset of slides resulted in no signal. A computer-assisted image analysis system was used for quantitative analysis of the immunostaining. The optical density and total area of immunoreactive cells were included. Only cells with their outline clearly defined by immunoparticles were counted as immunoreactive.

\section{Statistical analyses}

The data are presented as the mean \pm SD, and all computations were performed using SPSS [version 16.0]. One-way ANOVA and repeated-measures ANOVA were used. For all data, differences were considered significant at the level of $P<0.05$.

\section{Results}

\section{MWM performance}

Rats were subjected to MWM trials to investigate their spatial learning and memory ability. We found that, compared with the sham group, the cognitive ability in sham + DMSO group and in the sham + ACSF group were not affected by the surgery $(P>0.05$, Fig. 1$)$, and the cognitive abilities of the VD group, the VD + DMSO group, and the VD + ACSF group also did not significantly differ $(P>0.05$, Fig. 2$)$.

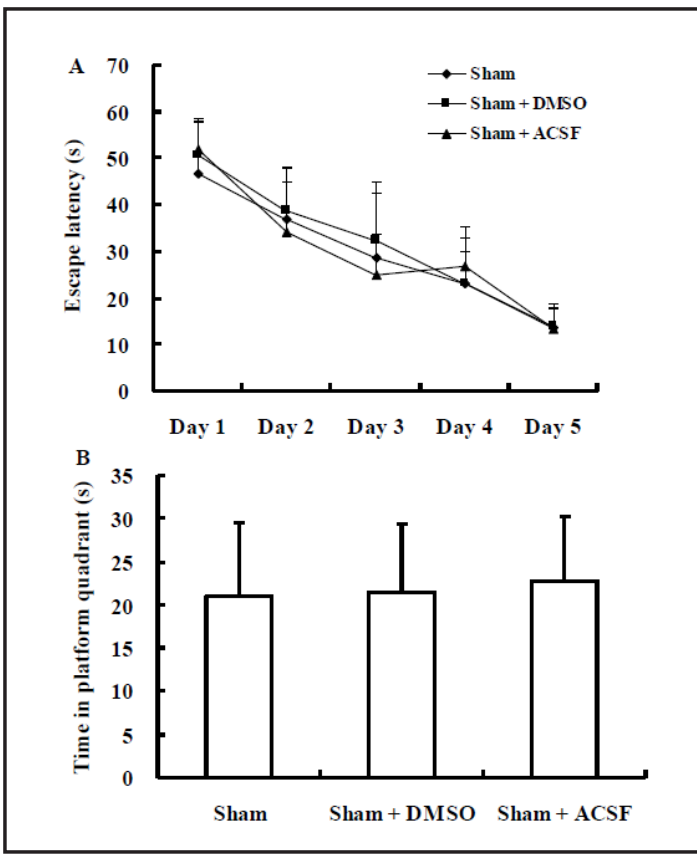

Fig. 1. Results of the MWM test. (A) Escape latencies over the 5-day training period in each of the three groups. (B) Time spent in the target quadrant during the probe trial (day 6). All data are expressed as the mean \pm SD ( $n=12$ each group).
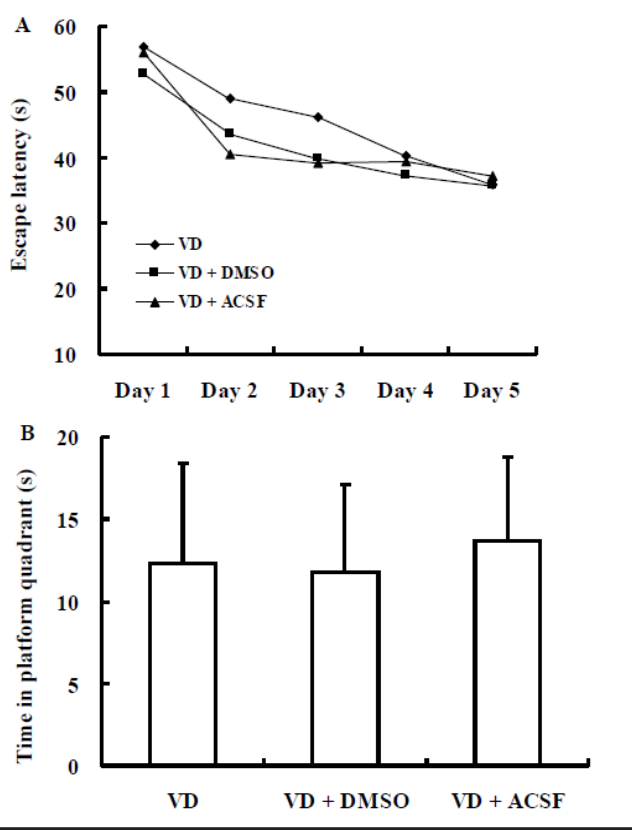

Fig. 2. Results of the MWM test. (A) Escape latencies over the 5-day training period in each of the three groups. (B) Time spent in the target quadrant during the probe trial (day 6). All data are expressed as the mean \pm SD $(n=12$ each group). 

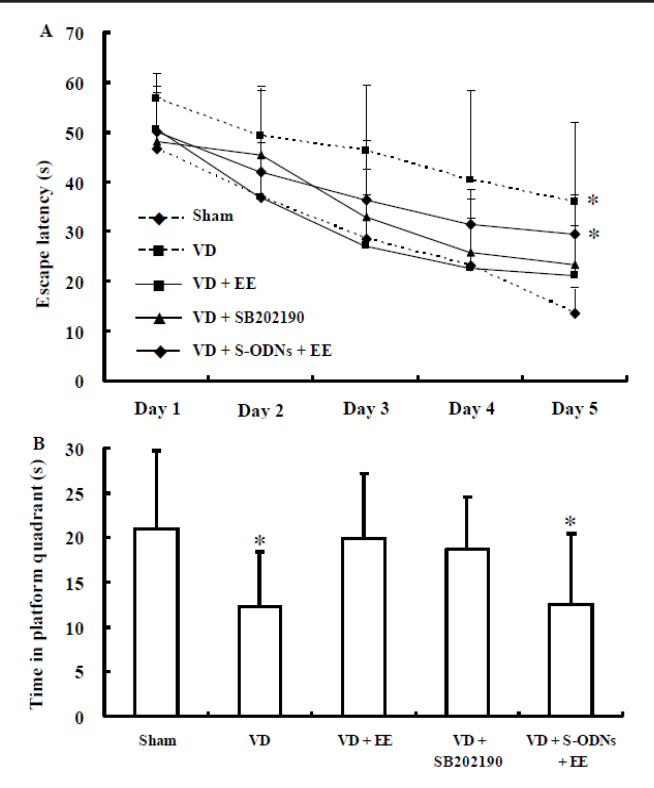

Fig. 3. The results of the MWM test. (A) Escape latency over the 5-day training period in each of the five groups. (B) Time spent in the target quadrant during the probe trial (day 6). All data are expressed as the mean $\pm \mathrm{SD}\left(\mathrm{n}=12\right.$ each group). ${ }^{*} P<0.05$ compared with the sham group, VD + EE group, and VD + SB203580 group.

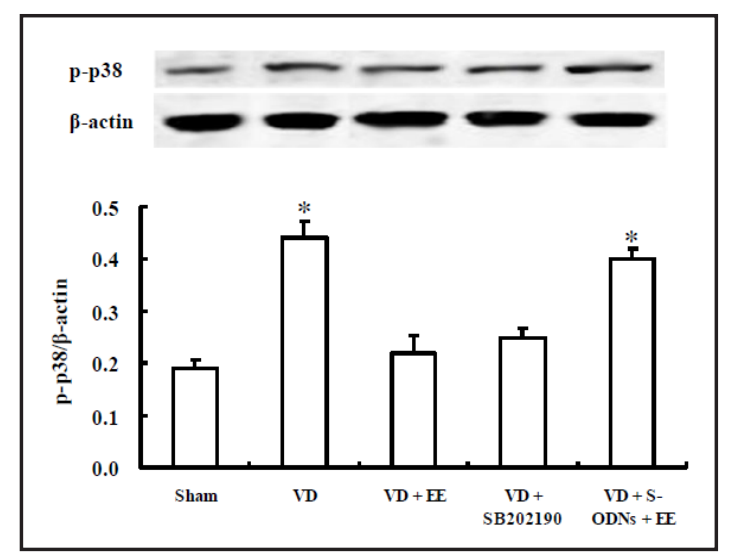

Fig. 4. Western blotting analysis of p-p38 protein expression in the rat CA1 hippocampus. The upper portion shows the immunoblot bands, and the lower portion is the quantitative presentation of the immunoblots with the ratio of the integral optical density (IOD) of the immunoblotting bands of p-p38 to $\beta$-actin (mean $\pm \mathrm{SD}, \mathrm{n}=5$ per group). ${ }^{*} P<0.05$ vs the sham group, VD + EE group, and VD + SB203580 group.

Figure 1 shows that hypoperfusion significantly increased the time of swimming until reaching the platform and decreased the time in the target quadrant compared with the sham rats $(P<0.05$, Fig. 3$)$. Interestingly, compared with the VD rats, the enriched environment induced recovery of the impaired cognitive performance of the hypoperfused rats $(P<0.05$, Fig. 3). Furthermore, application of the p38 MAPK S-ODNs to increase the effect of p38 MAPK significantly blocked the improvement in cognitive performance of the hypoperfused rats exposed to an EE compared with the EE + VD group $(P<0.05$, Fig. 3$)$. However, we found that the application of SB203580, p38 MAPK inhibitor, significantly ameliorated the cognitive impairment of rats after BCCAO compared with the VD group $(P<0.05$, Fig. 3$)$.

\section{Western Blot Analysis for $p$-p38 MAPK}

The levels of p-p38 MAPK protein were significantly upregulated in the VD control group compared with the sham group $(P<0.05$, Fig. 4), and exposure to an EE significantly downregulated p-p38 MAPK expression compared with the VD group $(P<0.05$, Fig. 4). Treatment with p38 MAPK S-ODNs effectively increased the downregulation of the p-p38 MAPK protein expression in the VD mice after exposure to an $\mathrm{EE}(P<0.05$, Fig. 4). In addition, the application of SB203580 resulted in the return of p-p38 MAPK protein expression to the levels of the sham group compared with the VD group $(P<0.05$, Fig. 4). Administration of vehicle in place of ACSF and DMSO had no effect on p-p38 MAPK protein expression levels in Sham + DMSO group, Sham + ACSF group, or VD + DMSO group, VD + ACSF group compared with the sham or VD rats, respectively ( $P>0.05$, Fig. 5 and 6 ).

\section{Immunohistochemistry for $p$ - $p 38 M A P K$}

Immunohistochemistry showed that p-p38 MAPK expression was markedly upregulated in the VD control group compared with the sham group $(P<0.05$, Fig. 7$)$, and exposure to an EE and treatment with SB203580 significantly downregulated p-p38 MAPK expression 


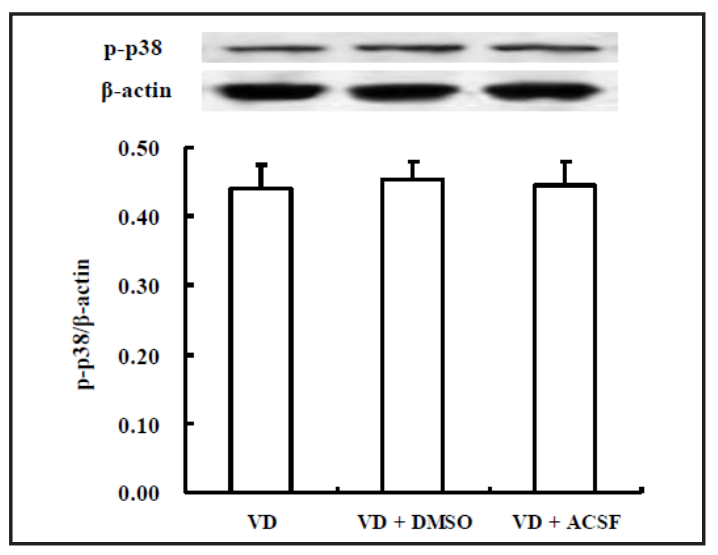

Fig. 5. Western blotting analysis of p-p38 protein expression in the rat CA1 hippocampus. The upper portion shows the immunoblot bands, and the lower portion is the quantitative presentation of the immunoblots with the ratio of the integral optical density (IOD) of the immunoblotting bands of p-p38 to $\beta$-actin (mean $\pm S D, n=5$ per group).

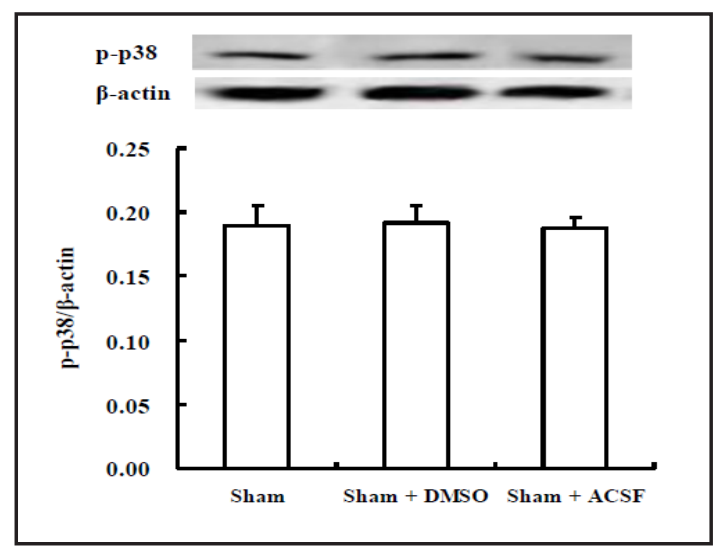

Fig. 6. Western blotting analysis of p-p38 protein expression in the rat CA1 hippocampus. The upper portion shows the immunoblot bands, and the lower portion is the quantitative presentation of the immunoblots with the ratio of the integral optical density (IOD) of the immunoblotting bands of p-p38 to $\beta$-actin (mean $\pm S D, n=5$ per group).
Fig. 7. Immunohistochemistry showing the expression of p-p38 MAPK in the rat CA1 hippocampal field. Panels in A are representative immunohistochemical staining photomicrographs of each group. Scale bar: $20 \mu \mathrm{m}$. Panels in B are quantitative presentations of the immunostaining with the optical density and the total area of the immunopositive cells in the CA1 hippocampus (mean $\pm \mathrm{SD}, \mathrm{n}=5$ per group). $* P<0.05$ vs the sham group, VD + EE group, and VD + SB203580 group.

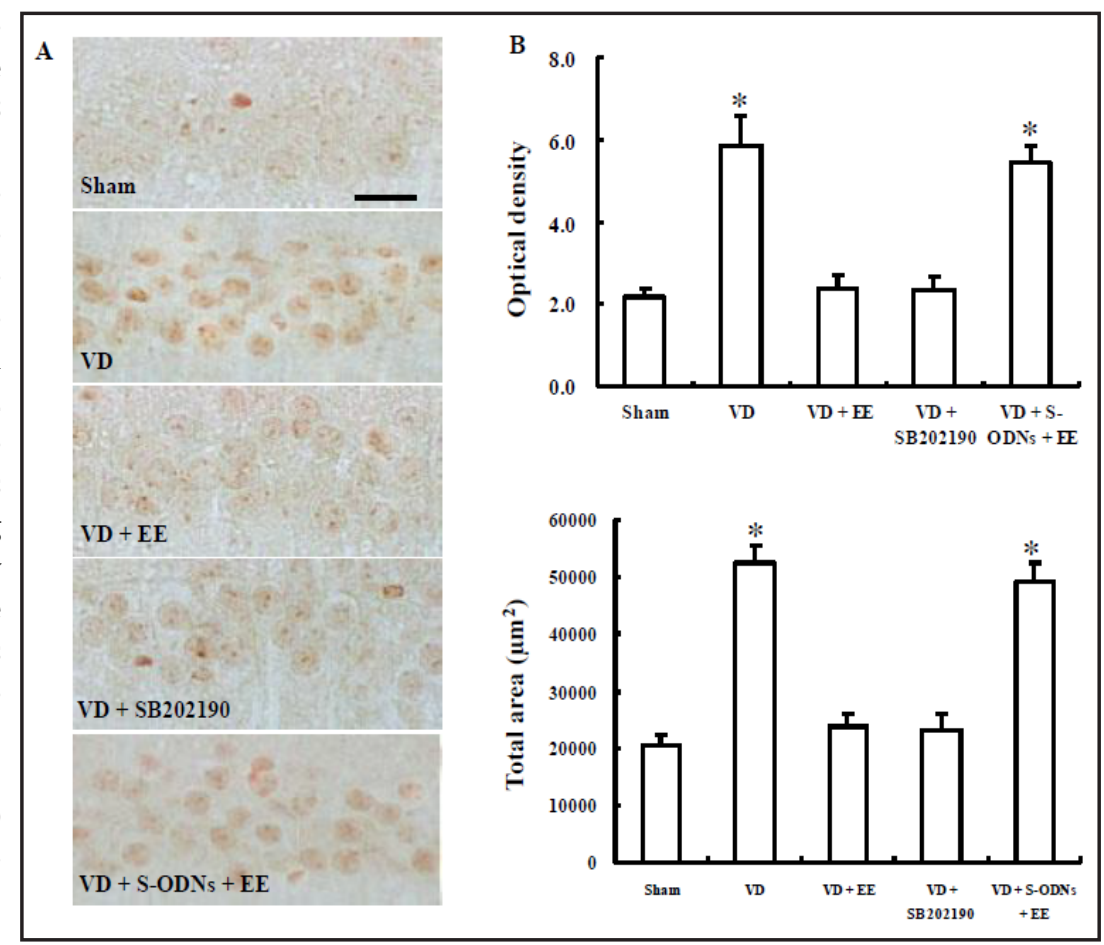

compared with the VD group ( $P<0.05$, Fig. 7). Treatment with p38 MAPK S-ODNs effectively increased the downregulation of the p-p38 MAPK expression in the VD mice after exposure to an $\mathrm{EE}(P<0.05$, Fig. 7).

\section{Discussion}

EE in the workplace stimulating verbal intelligence and executive functions may help to sustain a good cognitive functioning in humans [13]. In addition, studies with rodents found 


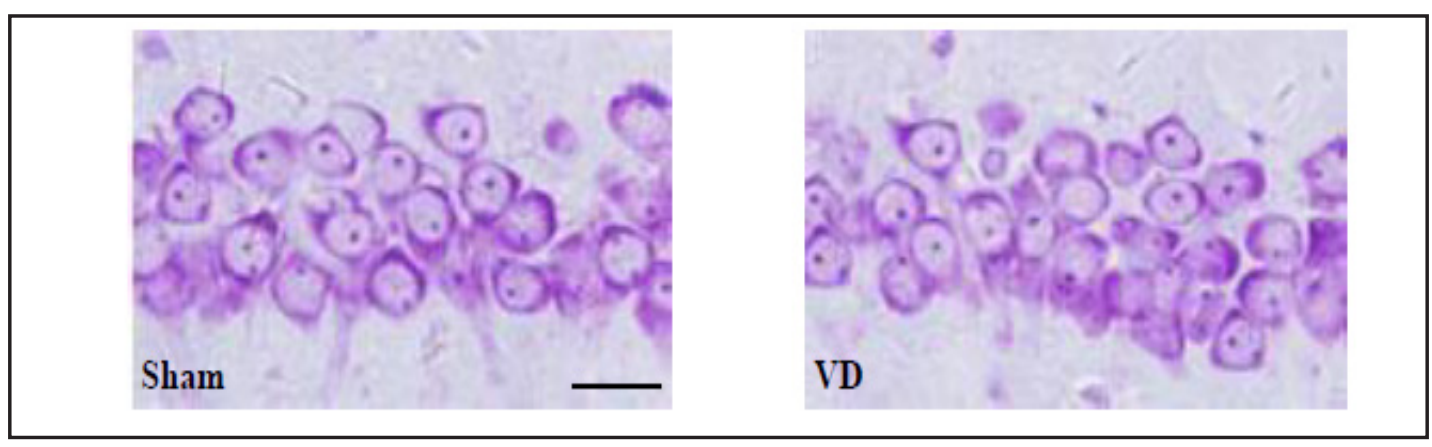

Fig. 8. Representative thionin-staining (method according to Sun et al.) [12] photomicrographs showing histologic changes of the hippocampal CA1 subfield in the sham and VD groups. Scale bar $=20 \mu \mathrm{m}$. The pyramidal neuronal density and histological grade of the CA1 area of the hippocampus were analyzed using the methods described by Kato et al. [22]. Briefly, the histological changes to CA1 were divided into 4 grades (histological grade) under a light microscope: grade 0, no cell death; grade 1, scattered cell death; grade 2, extensive cell death; and grade 3, almost complete cell death. The histological grade of the sham or VD group were assigned to grade 0 .

that EE exposure provides a "reserve"-like advantage that supports the preservation of spatial capabilities in aged rats [14] and in chronic cerebral hypoperfused rats [5, 15]. Our result also showed that EE could improve the learning and memory performance of VD model rats. A previous study concluded that EE applied to rodents results in regulatory mechanisms that give rise to significant beneficial effects at the molecular, cellular, and behavioral levels during brain development, particularly in the hippocampus [16]. BCCAO induces white matter and hippocampal damage, including microglial activation and proinflammatory mediator expression, and downregulation of the hyperphosphorylation of p38 MAPK signaling [17], and p38 MAPK signaling were also involved in other organs ischemia [18-20]. The results of the present study revealed an increase in p38 MAPK protein expression in VD rats, although the pathological of the hippocampal CA1 subfield in the sham and VD groups were not significantly different (Fig. 8). A blockade of the p38 MAPK signaling pathway by SB202190 following permanent BCCAO reduced apoptosis of hippocampal neurons and rescued spatial learning and memory deficits [21]. In our study, we found that application of the p38 MAPK inhibitor SB203580 also rescued the cognitive impairment of VD rats. After the SB203580 injection, the p38 MAPK protein was decreased parallel with the improvement in cognition of the VD rats. The improved cognition of the VD rats upon exposure to EE was inhibited after the application of p38 MAPK S-ODNs, accompanied by increased p38 MAPK in the hippocampus, suggested that the p38 MAPK signal participated the process of chronic cerebral hypoperfusion and the protection of EE. The above results of regulated p38 MAPK by EE are similar to the results reported by $\mathrm{Li}$ et al. that EE is involved in a gated signaling pathway, which rescues defective LTP induction in Ras-GRF knockout mice and consists of NMDA glutamate receptor activation of p38, a MAP kinase [8]. Based on the EE-gated signaling pathway, a thorough understanding of environmental control over specific cellsignaling cascades is needed to most effectively target signaling molecules for the treatment of human diseases. It is clear that the p38 MAPK signaling pathway exerts both beneficial and deleterious effects on the nervous system during chronic cerebral hypoperfusion. Identifying those conditions in which p38 MAPK contributes to neuronal damage may allow for the judicious use of EE for the treatment of VD; however, the mechanism and its upstream and downstream signal pathways remain to be studied.

In summary, these results suggest that $\mathrm{EE}$ ameliorates the spatial learning and memory impairments induced by BCCAO. This study also suggests that the cognitive impairment recovery induced by EE in the rat model of BCCAO is likely mediated by p38 MAPK signaling mechanisms. 


\section{Cellular Physiology Cell Physiol Biochem 2016;40:549-557

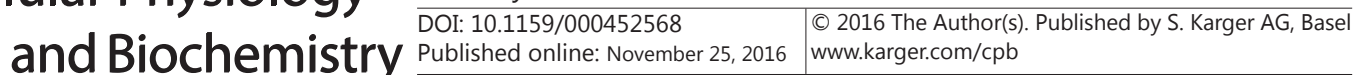 \\ Li et al.: p38 MAPK in the Improvement of Cognitive Deficits by EE}

\section{Acknowledgements}

This work was supported by the Tianjin Huanhu Hospital, and Natural Science Foundation of Tianjin Municipal Science and Technology Commission (13JCYBJC22700).

\section{Disclosure Statement}

The authors declare no conflicts of interest.

\section{References}

1 Venkat P, Chopp M, Chen J: Models and mechanisms of vascular dementia. Exp Neurol 2015;272:97-108.

2 Erdemli G, Xu YZ, Krnjevic K: Potassium conductance causing hyperpolarization of CA1 hippocampal neurons during hypoxia. J Neurophysiol 1998;80:2378-2390.

3 Fang M, Li J, Tiu SC, Zhang L, Wang M, Yew DT: N-methyl-D-aspartate receptor and apoptosis in Alzheimer's disease and multiinfarct dementia. J Neurosci Res 2005;81:269-274.

4 Matsumori Y, Hong SM, Fan Y, Kayama T, Hsu CY, Weinstein PR, Liu J: Enriched environment and spatial learning enhance hippocampal neurogenesis and salvages ischemic penumbra after focal cerebral ischemia. Neurobiol Dis 2006;22:187-198.

5 Bayat M, Sharifi MD, Haghani M, Shabani M: Enriched environment improves synaptic plasticity and cognitive deficiency in chronic cerebral hypoperfused rats. Brain Res Bull 2015;119:34-40.

6 Mora F, Segovia G, del Arco A: Aging, plasticity and environmental enrichment: structural changes and neurotransmitter dynamics in several areas of the brain. Brain Res Rev 2007;55:78-88.

7 Zhu H, Zhang J, Sun H, Zhang L, Liu H, Zeng X, Yang Y, Yao Z: An enriched environment reverses the synaptic plasticity deficit induced by chronic cerebral hypoperfusion. Neurosci Lett 2011;502:71-75.

8 Li S, Tian X, Hartley DM, Feig LA: The environment versus genetics in controlling the contribution of MAP kinases to synaptic plasticity. Curr Biol 2006;16:2303-2313.

9 Paxinos G WC: The rat brain in stereotaxic coordinates, Academic Press, New York, 1998.

10 Zhang M, Gong JX, Wang JL, Jiang MY, Li L, Hu YY, Qi J, Zhang LY, Zhao H, Cui X, Xian XH, Li WB: p38 MAPK Participates in the Mediation of GLT-1 Up-regulation During the Induction of Brain Ischemic Tolerance by Cerebral Ischemic Preconditioning. Mol Neurobiol DOI:10.1007/s12035-015-9652-x.

11 Zhang M, Li WB, Geng JX, Li QJ, Sun XC, Xian XH, Qi J, Li SQ: The upregulation of glial glutamate transporter-1 participates in the induction of brain ischemic tolerance in rats. J Cereb Blood Flow Metab 2007; 27:1352-1368.

12 Sun XC, Xian XH, Li WB, Li L, Yan CZ, Li QJ, Zhang M: Activation of p38 MAPK participates in brain ischemic tolerance induced by limb ischemic preconditioning by up-regulating HSP 70. Exp Neurol 2010;224:347355.

13 Then FS, Luck T, Luppa M, Konig HH, Angermeyer MC, Riedel-Heller SG: Differential effects of enriched environment at work on cognitive decline in old age. Neurology 2015;84:2169-2176.

14 Fuchs F, Cosquer B, Penazzi L, Mathis C, Kelche C, Majchrzak M, Barbelivien A: Exposure to an enriched environment up to middle age allows preservation of spatial memory capabilities in old age. Behav Brain Res 2016;299:1-5.

15 Zhang L, Zhang J, Sun H, Zhu H, Liu H, Yang Y: An enriched environment elevates corticosteroid receptor levels in the hippocampus and restores cognitive function in a rat model of chronic cerebral hypoperfusion. Pharmacol Biochem Behav 2013;103:693-700.

16 Grinan-Ferre C, Perez-Caceres D, Gutierrez-Zetina SM, Camins A, Palomera-Avalos V, Ortuno-Sahagun D, Rodrigo MT, Pallas M: Environmental Enrichment Improves Behavior, Cognition, and Brain Functional Markers in Young Senescence-Accelerated Prone Mice (SAMP8). Mol Neurobiol DOI:10.1007/s12035-0159210-6. 


\section{Cellular Physiology Cell Physiol Biochem 2016;40:549-557 \begin{tabular}{ll|l} 
DOI: 10.1159/000452568 & O 2016 The Author(s). Published by S. Karger AG, Basel \\
wwww.karger.com/cpb
\end{tabular} \\ Li et al.: p38 MAPK in the Improvement of Cognitive Deficits by EE}

17 Lee KM, Bang J, Kim BY, Lee IS, Han JS, Hwang BY, Jeon WK: Fructus mume alleviates chronic cerebral hypoperfusion-induced white matter and hippocampal damage via inhibition of inflammation and downregulation of TLR4 and p38 MAPK signaling. BMC Complement Altern Med 2015;15:125.

18 Chen Z, Zhang X, Liu Y, Liu Z: Morphine Postconditioning Protects against Reperfusion Injury via Inhibiting JNK/p38 MAPK and Mitochondrial Permeability Transition Pores Signaling Pathways. Cell Physiol Biochem 2016;39:61-70.

19 Xu Z, Yu J, Wu J, Qi F, Wang H, Wang Z: The Effects of Two Anesthetics, Propofol and Sevoflurane, on Liver Ischemia/Reperfusion Injury. Cell Physiol Biochem 2016;38:1631-1642.

20 Yang D, Zhang P, Wang T, Gao L, Qiao Z, Liang Y, Yu B: SalA attenuates ischemia/reperfusion-induced endothelial barrier dysfunction via down-regulation of VLDL receptor expression. Cell Physiol Biochem 2014;33:747-757.

21 Yang S, Zhou G, Liu H, Zhang B, Li J, Cui R, Du Y: Protective effects of p38 MAPK inhibitor SB202190 against hippocampal apoptosis and spatial learning and memory deficits in a rat model of vascular dementia. Biomed Res Int 2013;2013:215798.

22 Kato H, Liu Y, Araki T, Kogure K: Temporal profile of the effects of pretreatment with brief cerebral ischemia on the neuronal damage following secondary ischemic insult in the gerbil: cumulative damage and protective effects. Brain Res 1991;553:238-242. 